\title{
Effects of Amylose-To-Amylopectin Ratios on Binding Capacity of DDGS/Soy-Based Aquafeed Blends
}

\author{
Ferouz Ayadi $^{1}$, Kurt A. Rosentrater ${ }^{2}$, K. Muthukumarappan ${ }^{1}$, \& S. Kannadhason ${ }^{1}$ \\ ${ }^{1}$ Department of Agricultural and Biosystems Engineering, South Dakota State University, USA \\ ${ }^{2}$ Department of Agricultural and Biosystems Engineering, Iowa State University, USA \\ Correspondence: Kurt A. Rosentrater, Department of Agricultural and Biosystems Engineering, and Department \\ of Food Science and Human Nutrition, Iowa State University, Ames, IA 50011, USA. Tel: 1-515-294-4019. \\ E-mail: karosent@iastate.edu
}

Received: July 26, 2016

Accepted: August 15, 2016 Online Published: August 23, 2016

doi:10.5539/jfr.v5n5p43

URL: http://dx.doi.org/10.5539/jfr.v5n5p43

\begin{abstract}
Demands for seafood products are steadily increasing. Alternative protein sources are required to compensate for enormous amounts of fishmeal that is needed for global seafood production. Starch is a food polymer that can be added to fish feed formulations to enhance binding and expanding capabilities of extrudates. Floatability, a key factor for most aqua feeds, can be optimized by the addition of certain starch sources. Six ingredient blends with a similar protein content $(\sim 32.5 \%)$ containing two starch sources, Hylon VII (containing $70 \%$ amylose, $30 \%$ amylopectin) or Waxy I (containing 0\% amylose, 100\% amylopectin), 20\% distillers dried grain with solubles (DDGS), and 15, 25, and 35\% moisture content were used along with appropriate amounts of soybean meal, menhaden fishmeal, whey, vitamin and mineral mix to investigate nutritionally-balanced feeds for Nile tilapia (Oreochromis niloticus L.). The blends were processed using a laboratory single-screw extruder with varying temperature settings $\left(90-90-90^{\circ} \mathrm{C}, 100-120-120^{\circ} \mathrm{C}\right.$, and $\left.100-120-140^{\circ} \mathrm{C}\right)$, screw speeds $(100,120$, and $140 \mathrm{rpm})$, and length/diameter ratio $(3.4,6.6,9.2)$ of the die. Extensive analyses of expansion ratio (ER), unit density (UD), sinking velocity (SV), and pellet durability indices (PDI), water absorption (WAI) and water solubility indices (WSI) were conducted to evaluate the effects of the two starch sources on extrudate binding and floating capacity. By varying process conditions, significant differences $(\mathrm{P}>0.05)$ among the blends were detected for all extrudate physical properties. Significantly higher values for ER, UD, and PDI were achieved by using the Waxy I starch source, while values for SV and WAI decreased. For WSI no significant differences were detected. Increasing the moisture content from $15-35 \%$ resulted in a significant increase in ER, WAI, and PDI and a significant decrease in UD. WSI showed no clear pattern in changes. The impact of different amylopectin to amylose ratio, temperature and moisture content on extrudate stability, cohesion and physical properties was demonstrated in this study. All formulations yielded viable extrudates while the blends with the amylopectin as the sole source of starch resulted in higher quality extrudates.
\end{abstract}

Keywords: binding, corn, DDGS, expansion, extrusion, properties, soy, starch

\section{Introduction}

The world's hunger for seafood products has tremendously exploited and damaged global wild fish resources. Limited supply of seafood can only be regenerated by changing the global attitude towards utilizing nature's food resources and benefiting from aquaculture production. Moreover, aquaculture farming can take pressure of the dependency on wild fishery stocks by using alternative protein sources. In particular, carnivorous species require high amounts of fishmeal and fish oil in their diets. Furthermore, prices for fishmeal are so high that diets often represent $40-70 \%$ of aquaculture operating expenses (Thompson et al., 2008). Alternative protein sources are more cost effective and additionally, can support regional and local economies and can reduce environmental impact (Cheng and Hardy, 2004; Ayadi et al., 2009).

Numerous studies have examined alternative protein sources such as products from animal processing waste (e.g. meat and bone meal, poultry-by products, feather meal, etc.) and plant sources (e.g. soybean meal, corn, corn by-products, cottonseed meal, rapeseed meal, etc.). Soybean meal and corn are essential ingredients for fish feed formulations. Additional research is needed to investigate less expensive, more compatible, and sustainable sources that can replace fishmeal. Distillers dried grains with solubles (DDGS), a coproduct of grain 
fermentation from fuel ethanol or beverage alcohol production is another potential alternative to meet the protein requirements for fish. In contrast to the original grain, it contains about three times the amount of most nutrients due to the fermentation of starch to alcohol (Jacques et al., 2003; Klopfenstein et al., 2008). DDGS does not contain anti-nutritional factors that are commonly found in most plants (Lim et al., 2009). It has less phosphorus than fishmeal and may ultimately reduce the total phosphorus excreted into water and reduces water pollution (Cheng and Hardy, 2004). Changes in domestic energy policies and resulting growth of the fuel ethanol industry will subsequently increase quantities and availabilities of DDGS. DDGS is competitively priced and less expensive than other plant protein sources on a per unit protein basis (Bals et al., 2006; Lim et al., 2009), particularly when compared to soybean meal, which is the most commonly used substitute in aqua feeds.

Traditionally, DDGS has been fed to beef and dairy cattle (Klopfenstein et al., 2008; Schingoethe et al., 2009) and other livestock such as swine (Stein and Shurson, 2009) and poultry (Lumpkins et al., 2004). Since the late 1940s, DDGS has been integrated in fish feed at low inclusion levels (Thompson et al., 2008). In several studies, DDGS has been investigated for species such as Nile tilapia (Wu et al., 1996; Coyle et al., 2004; Lim et al., 2007), channel catfish (Webster et al., 1993; Robinson and Li, 2008; Lim et al., 2009), and rainbow trout (Cheng and Hardy, 2004) where 20-35\%, 30-40\%, and $22.5 \%$, respectively, could be included without adverse effects on growth performance and weight gain. These studies indicated that quality of feed that contain DDGS as a protein source, plays a key role in fish diets. Several studies demonstrated that processing conditions of fish feed is crucial to extrudate properties from both single and twin screw extrusion, especially when innovative materials are being utilized (Kannadhason et al., 2009a; Rosentrater et al., 2009b). Processing conditions impact pressure and shear forces within the extruder. Moisture content and screw speed have significant effects on extruder throughput, extrudate durability, and color (Chevanan et al., 2008). Feed moisture content, barrel temperature, extruder screw speed, die geometry, protein, and DDGS content significantly affect expansion ratio, sinking velocity, pellet durability index, extrudate color, mass flow rate, pressure at the die, and apparent viscosity (Kannadhason et al., 2009b; Rosentrater et al., 2009a; Chevanan et al., 2007, 2010). These studies ascertained a successful incorporation of up to 40\% and 60\% DDGS, while DDGS levels between 20-30\% are recommended for floating aquaculture feed (Kannadhason et al., 2009a). This is due to the moderately high fiber content of DDGS, which reduces the binding capacity of the blends (Webster et al., 1995). To assure a feed product with adequate floatability and binding capacities, binders such as cellulose or other less expensive starch sources can be added. Extrusion studies combining DDGS with different starch sources showed that cassava and tapioca starch, which have a high amylopectin proportion yielded the highest expansion ratios (Kannadhason et al., 2009a, 2009b; Rosentrater et al., 2009a, 2009b). Expansion, a key factor for floatability, is predominantly impacted by gelatinization of starch, which is governed by moisture content, temperature, pressure and shear forces in the extruder (Lai and Kokini, 1991). As an important food polymer, starch can be added to increase the viscosity, stability, and holding capacity of fat and water in fluids or semi-solid products (Hermansson and Svegmark, 1996). In aqua feeds, starch plays an important role for floatability based on its binding and expanding properties (Webster et al., 1995).

Starch is the reserve carbohydrate of plants and exists as water-insoluble heterogeneous granules with both amorphous and crystalline regions (Keetels et al., 1996a; Yu and Christie, 2005). It consists of the two major polysaccharides, amylose and amylopectin, of which amylopectin commonly makes up 70-80\%. The physical and biological properties of amylopectin are affected by a multiple branched structure formed by inter-chain linkage of every 20-25 glucose monomer. On the contrary, amylose content represents $20-30 \%$ of the content of most starches and forms longer linear glucose chains that are only lightly branched (Manners, 1989). Amylose and amylopectin have different functionalities and can be altered to desired property by plant breeding. Due to its crystalline order, amylopectin has stabilizing properties and shows higher storage stability whereas amylose tends to form gels and complexes. Waxy corn is a natural mutant without an amylose-producing enzyme that contains 100\% amylopectin (Hermansson and Svegmark, 1996). The amylose portion and the branching points of amylopectin form the amorphous region in the starch granules (Keetels et al., 1996; Yu and Christie, 2005). Hylon VII is a commercial available corn hybrid with high amylose content (70\%) that is used in the confectionary industry as a gelling and film-forming agent such as for jelly gum and batter coating (NSFI, 2008).

Gelatinization is not only determined by the molecular structure and chemical composition of starch granules, but heat treatment and available water also highly affect the processing of starch. During gelatinization, the granules swell and form a gel as the amylose and the amylopectin solubilize. This process generally occurs in presence of water at temperatures between $60-70^{\circ} \mathrm{C}$ (Hermansson and Svegmark, 1996). The swelling of the starch granules involves the separation of amylose and amylopectin (i.e., the leaching of amylose out of the granules) (Keetels et al., 1996b) and the destruction of the crystalline structure of the amylopectin (Yu and 
Christie, 2005).

Chinnaswamy and Hanna (1988) determined that expansion properties of starches were lowest at $70 \%$ and highest at 50\% amylose content, when investigating in starch sources with $0,25,50$, and $70 \%$ amylose proportions and were additionally influenced by temperature. Different starch blends expanded best at uniform moisture contents between 13-14\%.

Thermal processing of starch leads to degradation (debranching) and is affected by the moisture content. First, during heating at lower temperatures, long chains are cleaved followed by decomposition of the glucose rings. The second scission is affected by the moisture content: the higher the moisture content, the lower the temperature that is required to decompose the glucose ring (Liu et al., 2009).

Extrusion is a continuous process of short time cooking and forming of materials at relatively high pressure, heat, mechanical shear forces, and moisture. This involves order-disorder transition at different temperatures that impact the starch molecules size and shape, which includes starch gelatinization (under excess moisture) and protein denaturation (Lai and Kokini, 1991). Extrusion processing has been widely applied to produce digestible, palatable, durable, water stable, floating feed with high intake for fish.

Whey is the byproduct of the cheese making production. In studies with DDGS-based aquaculture feed, whey improved binding properties of extrudates and increased the pellet durability indices (Chevanan et al., 2009).

This study continued the research previously done by Kannadhason et al. (2009a, 2009b) and Rosentrater et al. (2009a, 2009b) to examine the effect of starch as a binder. Thus, the objectives of this study were: 1) to produce viable extruded feed for juvenile Nile tilapia using DDGS/soy as an alternative protein source in combination with two different starch sources, and 2) to examine the effects of different amylose/amylopectin ratios, various levels of feed moisture content, extruder die temperature, screw speed, and length-to-diameter (L/D) ratio, on the resulting physical properties of the extrudates and on various processing parameters.

\section{Materials and Methods}

\subsection{Feed Blend Preparation}

Six ingredient blends suitable for Nile tilapia fish were formulated to a target protein level of $32.5 \% \mathrm{db}$ using $20 \%$ DDGS and 48\% soybean meal, and 15\% corn [either Hylon VII (containing 70\% amylose, 30\% amylopectin) or Waxy I (containing 0\% amylose, 100\% amylopectin)] as the starch source, and three moisture contents $(15,25$, and $35 \%$ ), along with appropriate quantities of soybean meal, menhaden fishmeal, whey, vitamin and mineral mix (Table 1) to prepare nutritionally-balanced diets for Nile tilapia. Hylon VII corn was obtained from National Starch Food Innovation (Bridgewater, NJ), while Waxy No. 1 corn was from Tate and Lyle (Decatur, IL). DDGS was provided by Dakota Ethanol LLC (Wentworth, SD) and soybean meal was from Dakotaland Feeds Inc., LLC (Huron, SD). All were ground with a Wiley Mill (Model 4, Thomas Scientific, Swedesboro, NJ) to a powder with an average particle size of approximately $500 \mu \mathrm{m}$. Menhaden fishmeal was purchased from Consumers Supply Distributing Co. (North Sioux City, SD); vitamin C was from DSM Nutritional Products France SAS (Village-Neuf, France); whey, vitamin mix and mineral mix were obtained from Lortscher Agri Service, Inc. (Bern, KS). The ingredients were mixed in a laboratory-scale mixer (N50 mixer, Hobart Co., Troy, $\mathrm{OH})$ for $10 \mathrm{~min}$ and adjusted with adequate amounts of water to the target moisture content.

Table 1. Ingredient components ( $\mathrm{g} / 100 \mathrm{~g}$, dry basis) in the feed blends used in the study.

\begin{tabular}{lc}
\hline & $\begin{array}{l}\text { Dry weight of } \\
\text { ingredients }(\mathrm{g} / 100 \mathrm{~g})\end{array}$ \\
\hline DDGS $^{1}$ & 20 \\
Soybean meal $^{2}$ & 48 \\
Corn $^{3}$ & 15 \\
${\text { Menhaden fish } \text { meal }^{4}}^{4}$ & 9 \\
Whey $^{5}$ & 5 \\
Vitamin/mineral premix $^{6}$ & 2 \\
${\text { Vitamin C } \text { mix }^{7}}^{\text {Total }}$ & 1 \\
\hline
\end{tabular}

1 Dakota Ethanol Plant (Wentworth, SD)

2 Dakotaland Feeds Inc., LLC (Huron, SD)

3 Hylon VII: National Starch Food Innovation (Bridgewater, NJ); Waxy No. 1: Tate and Lyle (Decatur, IL)

4 Consumers Supply Distribution Co. (North Sioux City, SD)

5 Animal feed dried whey, Midor Ltd (Elroy, WI)

6 Lortscher Agri Service, Inc. (Bern, KS)

7 DSM Nutritional Products France SAS (Village-Neuf, France) 


\subsection{Extrusion Processing}

All blends were processed using a single screw extruder (Brabender Plasti-Corder, Model PL 2000, South Hackensack, NJ) which had a compression ratio of 3:1, with a screw length-to-diameter ratio of 20:1, and a barrel length of $317.5 \mathrm{~mm}$. The center of the die assembly was conical, and tapered from an initial diameter of $6.0 \mathrm{~mm}$ to the die diameters of $1.8,2.6$, and $2.8 \mathrm{~mm}$ (length-to-diameter ratios of $9.2,6.6$ and $3.4 \mathrm{~mm}$, respectively) at the exiting of the extruder. The screw speed and the barrel temperature were monitored by a computer that was attached to the extruder. The temperature of the feed, transition and die zone were adjusted with external band heaters to three temperature profiles (feed-transition-die sections) in the barrel $\left(90-90-90^{\circ} \mathrm{C}\right.$, $100-120-120^{\circ} \mathrm{C}$, and $100-120-140^{\circ} \mathrm{C}$, respectively). A $7.5 \mathrm{HP}(5.5 \mathrm{~kW})$ motor was connected to the extruder to control the screw speed from 0 to $210 \mathrm{rpm}(22 \mathrm{rad} / \mathrm{s})$.

The raw blends were funneled into the extruder manually in constant quantities to avoid jamming at the opening to the barrel. The mass flow rate (MFR) was determined by collecting extrudate samples at $30 \mathrm{~s}$ intervals during extrusion, and then weighing the collected amount on an electronic balance (PB 5001, Mettler Toledo, Switzerland).

\subsection{Extrudate Properties}

After processing, the extrudates were dried for $24 \mathrm{~h}$ at room temperature $\left(20 \pm 1^{\circ} \mathrm{C}\right)$ and triplicates $(\mathrm{n}=3)$ were then subjected to an extensive physical property analysis of expansion ratio (-), unit density $\left(\mathrm{kg} / \mathrm{m}^{3}\right)$, water absorption index (-), water solubility index (\%), and pellet durability index $(\%)$.

Expansion ratio (ER)

Radial expansion ratio has been determined as the ratio of the diameter of the dry extrudate that was measured with a digital calliper (Digimatic calliper, Model No: CD-6"C, Mitutoyo Corp., Tokyo, Japan), to the diameter of the die nozzle $(1.8,2.6$, and $2.8 \mathrm{~mm}$, respectively). The results were displayed as the mean of ten $(\mathrm{n}=10)$ measurements.

Unit density (UD)

Extrudate at approximately sizes of $25.4 \mathrm{~mm}$ were weighed on an analytical balance (Adventurer ${ }^{\mathrm{TM}}$, Item No: AR 1140, Ohaus Corp. Pine Brook, NJ), and then measured with a digital calliper (Digimatic calliper, Model No: CD-6"C, Mitutoyo Corp., Tokyo, Japan) to determine their diameter. According to Rosentrater et al. (2005) the unit density $\left(\mathrm{UD}, \mathrm{kg} / \mathrm{m}^{3}\right)$ was calculated as the ratio of the mass $\mathrm{M}(\mathrm{kg})$ to the volume $\mathrm{V}\left(\mathrm{m}^{3}\right)$ of each measured and weighed extrudate sample, assuming a cylindrical shape for each extrudate:

$$
\mathrm{UD}=\frac{M}{V}
$$

Sinking velocity (SV)

Sinking velocity was determined following Himadri et al. (1993) by measuring the time an extrudate of approximately $25.4 \mathrm{~mm}$ length needed to reach the bottom of a $2 \mathrm{~L}$ measuring cylinder filled with distilled water. The total distance $(0.415 \mathrm{~m})$ required for the time yielded the sinking viscosity $(\mathrm{m} / \mathrm{s})$.

Water absorption and water solubility index (WAI and WSI)

The procedure described by Anderson et al. (1969) was used to measure water absorption index (WAI) and water solubility index. These parameters were used to quantify binding capacity of the extrudates. Approximately $2.5 \mathrm{~g}$ of finely ground extrudate sample $(150 \mu \mathrm{m})$ was suspended in $30 \mathrm{~mL}$ of distilled water in a tarred centrifuge tube of $50 \mathrm{~mL}$ and placed in a laboratory oven (Thelco precision, Jovan Inc., Wincester, VA) at $30^{\circ} \mathrm{C}$. At intervals, it was then stirred intermittently for a period of $30 \mathrm{~min}$. Subsequently, the centrifuge tube was centrifuged and the supernatant was decanted into an aluminum dish, which was placed in the oven for $2 \mathrm{~h}$ at $135^{\circ} \mathrm{C}$ (AACC method $44-19,2000)$ and then desiccated for $20 \mathrm{~min}$. The dry solids and the gel mass were weighed. WAI (-) was determined as the ratio of gel mass to the original sample mass (2.5 g ground sample). WSI (\%) was calculated as the ratio of the mass of the dry solids (recovered from evaporation of the supernatant from the WAI test) to the original sample mass. WAI is the gel weight received per gram of dry sample, whereas WSI quantifies the starch portion that remains in the water phase when exposed to water. The better the binding capacity, the lower the leaching losses to water.

Pellet durability (PDI)

The pellet durability index was quantified according to Method S269.4 (ASAE, 2004). To separate initial fines from each blend, about $100 \mathrm{~g}$ of extrudates were manually sieved (U.S.A. standard testing, ASTM E-11 specification, Daigger, Vernon Hills, IL) for about $10 \mathrm{~s}$, and then tumbled in a pellet durability tester (model PDT-110, Seedburo Equipment Company, Chicago, IL) for $10 \mathrm{~min}$. Afterwards, the samples were again sieved for about $10 \mathrm{~s}$, and then weighed on an electronic balance (Explorer Pro, Model: EP4102, Ohaus, Pine Brook, $\mathrm{NJ})$. PDI was calculated as: 


$$
\text { PDI }=\left(\frac{M_{a}}{M_{b}}\right) \times 100
$$

where $\mathrm{M}_{\mathrm{a}}$ was the mass $(\mathrm{g})$ after tumbling and $\mathrm{M}_{\mathrm{b}}$ was the sample mass $(\mathrm{g})$ before tumbling.

\subsection{Experimental Design}

Six blends were prepared with 20\% DDGS, one starch sources (Waxy I or Hylon VII), three levels of moisture content $(15,25$, and $35 \%)$, with a constant protein content $(32.5 \%)$. During processing, three temperature profiles were adjusted in the barrel $\left(90-90-90^{\circ} \mathrm{C}, 100-120-120^{\circ} \mathrm{C}\right.$, and $100-120-140^{\circ} \mathrm{C}$, respectively), and were referred to as temperatures of 100,120 , and $140^{\circ} \mathrm{C}$, respectively, at three screw speeds $(100,130$, and $160 \mathrm{rpm}$, respectively), and three levels of die geometry with various nozzle length-to-diameter (L/D) ratios (9.2, 6.6 and $3.4 \mathrm{~mm}$, respectively).

This resulted in $3 \times 3 \times 3 \times 2=162$ total treatment combinations (Table 2). Triplicates $(n=3)$ were measured for most physical properties (i.e., dependent variables) for each treatment combination, except for pellet durability index, which was only measured in duplicate.

The data were then analyzed with Proc GLM to determine the main, interaction and treatment combination effects using SAS software (SAS Institute, Cary, NC) with a Type I error rate $(\alpha)$ of 0.05 .

Table 2. Experimental design.*

\begin{tabular}{|c|c|c|c|c|c|c|c|c|c|c|c|c|c|c|c|c|c|}
\hline $\begin{array}{l}\text { Treat- } \\
\text { ment }\end{array}$ & $\begin{array}{l}\text { Starch } \\
\text { type }\end{array}$ & $\begin{array}{c}\text { Moisture } \\
\text { content }\end{array}$ & Tdie & $\begin{array}{l}\text { Screw } \\
\text { speed }\end{array}$ & $\begin{array}{l}\mathrm{Die} \\
\mathrm{L} / \mathrm{D}\end{array}$ & $\begin{array}{c}\text { Treat- } \\
\text { ment }\end{array}$ & $\begin{array}{c}\text { Starch } \\
\text { type }\end{array}$ & $\begin{array}{c}\text { Moisture } \\
\text { content }\end{array}$ & Tdie & $\begin{array}{l}\text { Screw } \\
\text { speed }\end{array}$ & $\begin{array}{l}\mathrm{Die} \\
\mathrm{L} / \mathrm{D}\end{array}$ & $\begin{array}{l}\text { Treat- } \\
\text { ment }\end{array}$ & $\begin{array}{c}\text { Starch } \\
\text { type }\end{array}$ & $\begin{array}{c}\text { Moisture } \\
\text { content }\end{array}$ & Tdie & $\begin{array}{l}\text { Screw } \\
\text { speed }\end{array}$ & $\begin{array}{l}\mathrm{Die} \\
\mathrm{L} / \mathrm{D}\end{array}$ \\
\hline & & $(\% \mathrm{db})$ & $\left({ }^{\circ} \mathrm{C}\right)$ & (rpm) & $(-)$ & & & $(\% \mathrm{db})$ & $\left({ }^{\circ} \mathrm{C}\right)$ & $(\mathrm{rpm})$ & $(-)$ & & & $(\% \mathrm{db})$ & $\left({ }^{\circ} \mathrm{C}\right)$ & (rpm) & $(-)$ \\
\hline 1 & Hylon VII & 15 & 100 & 100 & 3.4 & 55 & & 35 & 100 & 100 & 3.4 & 109 & & 25 & 100 & 100 & 3.4 \\
\hline 2 & & & & & 6.6 & 56 & & & & & 6.6 & 110 & & & & & 6.6 \\
\hline 4 & & & & 130 & 3.4 & 58 & & & & 130 & 3.4 & 112 & & & & 130 & 3.4 \\
\hline 5 & & & & & 6.6 & 59 & & & & & 6.6 & 113 & & & & & 6.6 \\
\hline 6 & & & & & 9.2 & 60 & & & & & 9.2 & 114 & & & & & 9.2 \\
\hline 7 & & & & 160 & 3.4 & 61 & & & & 160 & 3.4 & 115 & & & & 160 & 3.4 \\
\hline 9 & & & & & 9.2 & 63 & & & & & 9.2 & 117 & & & & & 9.2 \\
\hline 10 & & & 120 & 100 & 3.4 & 64 & & & 120 & 100 & 3.4 & 118 & & & 120 & 100 & 3.4 \\
\hline 11 & & & & & 6.6 & 65 & & & & & 6.6 & 119 & & & & & 6.6 \\
\hline 12 & & & & & 9.2 & 66 & & & & & 9.2 & 120 & & & & & 9.2 \\
\hline 13 & & & & 130 & 3.4 & 67 & & & & 130 & 3.4 & 121 & & & & 130 & 3.4 \\
\hline 14 & & & & & 6.6 & 68 & & & & & 6.6 & 122 & & & & & 6.6 \\
\hline 15 & & & & & 9.2 & 69 & & & & & 9.2 & 123 & & & & & 9.2 \\
\hline 16 & & & & 160 & 3.4 & 70 & & & & 160 & 3.4 & 124 & & & & 160 & 3.4 \\
\hline 17 & & & & & 6.6 & 71 & & & & & 6.6 & 125 & & & & & 6.6 \\
\hline 18 & & & & & 9.2 & 72 & & & & & 9.2 & 126 & & & & & 9.2 \\
\hline 20 & & & & & 6.6 & 74 & & & & & 6.6 & 128 & & & & & 6.6 \\
\hline 21 & & & & & 9.2 & 75 & & & & & 9.2 & 129 & & & & & 9.2 \\
\hline 22 & & & & 130 & 3.4 & 76 & & & & 130 & 3.4 & 130 & & & & 130 & 3.4 \\
\hline 23 & & & & & 6.6 & 77 & & & & & 6.6 & 131 & & & & & 6.6 \\
\hline 24 & & & & & 9.2 & 78 & & & & & 9.2 & 132 & & & & & 9.2 \\
\hline 25 & & & & 160 & 3.4 & 79 & & & & 160 & 3.4 & 133 & & & & 160 & 3.4 \\
\hline 26 & & & & & 6.6 & 80 & & & & & 6.6 & 134 & & & & & 6.6 \\
\hline 27 & & & & & 9.2 & 81 & & & & & 9.2 & 135 & & & & & 9.2 \\
\hline 28 & & 25 & 100 & 100 & 3.4 & 82 & Waxy I & 15 & 100 & 100 & 3.4 & 136 & & 35 & 100 & 100 & 3.4 \\
\hline 29 & & & & & 6.6 & 83 & & & & & 6.6 & 137 & & & & & 6.6 \\
\hline 30 & & & & & 9.2 & 84 & & & & & 9.2 & 138 & & & & & 9.2 \\
\hline 31 & & & & 130 & 3.4 & 85 & & & & 130 & 3.4 & 139 & & & & 130 & 3.4 \\
\hline 32 & & & & & 6.6 & 86 & & & & & 6.6 & 140 & & & & & 6.6 \\
\hline 33 & & & & & 9.2 & 87 & & & & & 9.2 & 141 & & & & & 9.2 \\
\hline 34 & & & & 160 & 3.4 & 88 & & & & 160 & 3.4 & 142 & & & & 160 & 3.4 \\
\hline 35 & & & & & 6.6 & 89 & & & & & 6.6 & 143 & & & & & 6.6 \\
\hline 36 & & & & & 9.2 & 90 & & & & & 9.2 & 144 & & & & & 9.2 \\
\hline 37 & & & 120 & 100 & 3.4 & 91 & & & 120 & 100 & 3.4 & 145 & & & 120 & 100 & 3.4 \\
\hline 38 & & & & & 6.6 & 92 & & & & & 6.6 & 146 & & & & & 6.6 \\
\hline 39 & & & & & 9.2 & 93 & & & & & 9.2 & 147 & & & & & 9.2 \\
\hline 43 & & & & 160 & 3.4 & 97 & & & & 160 & 3.4 & 151 & & & & 160 & 3.4 \\
\hline 44 & & & & & 6.6 & 98 & & & & & 6.6 & 152 & & & & & 6.6 \\
\hline 45 & & & & & 9.2 & 99 & & & & & 9.2 & 153 & & & & & 9.2 \\
\hline 46 & & & 140 & 100 & 3.4 & 100 & & & 140 & 100 & 3.4 & 154 & & & 140 & 100 & 3.4 \\
\hline 47 & & & & & 6.6 & 101 & & & & & 6.6 & 155 & & & & & 6.6 \\
\hline 48 & & & & & 9.2 & 102 & & & & & 9.2 & 156 & & & & & 9.2 \\
\hline 49 & & & & 130 & 3.4 & 103 & & & & 130 & 3.4 & 157 & & & & 130 & 3.4 \\
\hline 50 & & & & & 6.6 & 104 & & & & & 6.6 & 158 & & & & & 6.6 \\
\hline 51 & & & & & 9.2 & 105 & & & & & 9.2 & 159 & & & & & 9.2 \\
\hline 52 & & & & 160 & 3.4 & 106 & & & & 160 & 3.4 & 160 & & & & 160 & 3.4 \\
\hline 53 & & & & & 6.6 & 107 & & & & & 6.6 & 161 & & & & & 6.6 \\
\hline 54 & & & & & 9.2 & 108 & & & & & 9.2 & 162 & & & & & 9.2 \\
\hline
\end{tabular}

* The experimental design consisted of 2 (starch sources) x 3 (moisture contents) x 3 (die temperatures) x 3 (screw speeds) x $3($ die $\mathrm{L} / \mathrm{D})=162$ total treatment combinations. Tdie is temperature of the die, die L/D is length-to-diameter ratio of the die. 


\section{Results and Discussion}

Tables 3, 4, and 5 summarize the main treatment effects and interaction effects of the two different amylose/amylopectin ratios, different moisture contents, temperature profiles, screw speeds, and L/D ratios of the die. The different starch sources had significant effects on all extrudate properties at $\alpha=0.05$. Likewise, the moisture contents $(15,25$, and $25 \%)$ significantly affected all tested parameters, except for WSI, as well as increasing the screw speed from 100 to 130 and $160 \mathrm{rpm}$ resulted in significant differences, except for UD and PDI. Table 3 provides main effects resulting from considering both starches simultaneously; Table 4 also provides the main effects, but type of starch is used as a blocking factor. It can readily be seen that the behaviours of the dependent variables, when considering each starch separately, were similar to the behaviours when the starches were considered together (i.e., Figures 1 and 2).

Table 3. Main effects due to starch source, moisture content, die temperature, screw speed, and die L/D on resulting extrudate physical properties $(n=3, \alpha=0.05)$.*

\begin{tabular}{|c|c|c|c|c|c|c|c|}
\hline Parameter & Levels & $\begin{array}{c}\text { ER } \\
(-)\end{array}$ & $\begin{array}{c}\mathrm{UD} \\
\left(\mathrm{kg} / \mathrm{m}^{3}\right)\end{array}$ & $\begin{array}{l}\mathrm{SV} \\
(\mathrm{m} / \mathrm{s})\end{array}$ & $\begin{array}{c}\text { WAI } \\
(-)\end{array}$ & $\begin{array}{c}\text { WSI } \\
(-)\end{array}$ & $\begin{array}{l}\text { PDI } \\
(\%)\end{array}$ \\
\hline \multirow[t]{2}{*}{ Starch source } & Hylon VII & $\begin{array}{l}1.17 \mathrm{a} \\
(0.06)\end{array}$ & $\begin{array}{l}0.97 \mathrm{a} \\
(0.08)\end{array}$ & $\begin{array}{l}0.076 \mathrm{a} \\
(0.012)\end{array}$ & $\begin{array}{l}3.04 \mathrm{a} \\
(0.29)\end{array}$ & $\begin{array}{l}18.17 \mathrm{a} \\
(2.56)\end{array}$ & $\begin{array}{l}92.85 \mathrm{a} \\
(3.53)\end{array}$ \\
\hline & Waxy I & $\begin{array}{l}1.20 \mathrm{~b} \\
(0.09)\end{array}$ & $\begin{array}{l}0.99 \mathrm{~b} \\
(0.10)\end{array}$ & $\begin{array}{l}0.068 b \\
(0.024)\end{array}$ & $\begin{array}{l}2.83 \mathrm{~b} \\
(0.30)\end{array}$ & $\begin{array}{l}18.27 \mathrm{a} \\
(2.27)\end{array}$ & $\begin{array}{r}95.98 \mathrm{~b} \\
(1.64)\end{array}$ \\
\hline \multirow[t]{3}{*}{$\begin{array}{l}\text { Moisture content } \\
(\% \mathrm{db})\end{array}$} & 15 & $\begin{array}{l}1.22 \mathrm{a} \\
(0.06)\end{array}$ & $\begin{array}{l}1.02 \mathrm{a} \\
(0.05)\end{array}$ & $\begin{array}{l}0.081 \mathrm{a} \\
(0.009)\end{array}$ & $\begin{array}{l}2.86 \mathrm{a} \\
(0.29)\end{array}$ & $\begin{array}{l}18.42 \mathrm{a} \\
(2.09)\end{array}$ & $\begin{array}{l}93.98 \mathrm{a} \\
(4.59)\end{array}$ \\
\hline & 25 & $\begin{array}{l}1.20 \mathrm{~b} \\
(0.08)\end{array}$ & $\begin{array}{l}0.94 \mathrm{~b} \\
(0.11)\end{array}$ & $\begin{array}{l}0.065 c \\
(0.026)\end{array}$ & $\begin{array}{l}2.93 \mathrm{~b} \\
(0.30)\end{array}$ & $\begin{array}{l}17.67 \mathrm{~b} \\
(3.07)\end{array}$ & $\begin{array}{l}93.17 \mathrm{~b} \\
(2.22)\end{array}$ \\
\hline & 35 & $\begin{array}{l}1.14 \mathrm{c} \\
(0.07)\end{array}$ & $\begin{array}{l}0.97 \mathrm{c} \\
(0.10)\end{array}$ & $\begin{array}{l}0.069 b \\
(0.018)\end{array}$ & $\begin{array}{l}3.02 \mathrm{c} \\
(0.31)\end{array}$ & $\begin{array}{l}18.55 \mathrm{a} \\
(2.23)\end{array}$ & $\begin{array}{l}95.54 \mathrm{c} \\
(2.05)\end{array}$ \\
\hline \multirow[t]{3}{*}{$\begin{array}{l}\text { Temperature of } \\
\text { die }\left({ }^{\circ} \mathrm{C}\right)\end{array}$} & 100 & $\begin{array}{l}1.17 \mathrm{a} \\
(0.07)\end{array}$ & $\begin{array}{l}1.04 \mathrm{a} \\
(0.08)\end{array}$ & $\begin{array}{l}0.082 \mathrm{a} \\
(0.009)\end{array}$ & $\begin{array}{l}2.88 \mathrm{a} \\
(0.26)\end{array}$ & $\begin{array}{l}18.12 \mathrm{a} \\
(2.33)\end{array}$ & $\begin{array}{l}95.13 \mathrm{a} \\
(2.64)\end{array}$ \\
\hline & 120 & $\begin{array}{l}1.19 \mathrm{~b} \\
(0.08)\end{array}$ & $\begin{array}{l}0.98 \mathrm{~b} \\
(0.07)\end{array}$ & $\begin{array}{l}0.072 b \\
(0.015)\end{array}$ & $\begin{array}{l}2.89 \mathrm{a} \\
(0.30)\end{array}$ & $\begin{array}{l}18.61 \mathrm{~b} \\
(2.57)\end{array}$ & $\begin{array}{l}94.65 \mathrm{~b} \\
(1.81)\end{array}$ \\
\hline & 140 & $\begin{array}{l}1.19 \mathrm{~b} \\
(0.08) \\
\end{array}$ & $\begin{array}{l}0.92 \mathrm{c} \\
(0.09)\end{array}$ & $\begin{array}{l}0.061 \mathrm{c} \\
(0.026)\end{array}$ & $\begin{array}{l}3.04 \mathrm{~b} \\
(0.34)\end{array}$ & $\begin{array}{l}17.93 a \\
(2.60)\end{array}$ & $\begin{array}{l}92.98 \mathrm{c} \\
(4.53)\end{array}$ \\
\hline \multirow[t]{3}{*}{$\begin{array}{l}\text { Screw speed } \\
(\mathrm{rpm})\end{array}$} & 100 & $\begin{array}{l}1.17 \mathrm{a} \\
(0.07)\end{array}$ & $\begin{array}{l}0.99 \mathrm{~b} \\
(0.10)\end{array}$ & $\begin{array}{l}0.073 a \\
(0.016)\end{array}$ & $\begin{array}{l}2.94 \mathrm{a} \\
(0.32)\end{array}$ & $\begin{array}{l}18.61 \mathrm{a} \\
(2.83)\end{array}$ & $\begin{array}{l}93.78 \mathrm{a} \\
(4.45)\end{array}$ \\
\hline & 130 & $\begin{array}{l}1.19 \mathrm{~b} \\
(0.07)\end{array}$ & $\begin{array}{c}0.98 \mathrm{ab} \\
(0.09)\end{array}$ & $\begin{array}{l}0.074 b \\
(0.018)\end{array}$ & $\begin{array}{l}2.89 \mathrm{~b} \\
(0.30)\end{array}$ & $\begin{array}{l}18.35 \mathrm{~b} \\
(2.55)\end{array}$ & $\begin{array}{l}94.58 \mathrm{~b} \\
(2.53)\end{array}$ \\
\hline & 160 & $\begin{array}{l}1.20 \mathrm{c} \\
(0.09) \\
\end{array}$ & $\begin{array}{l}0.97 \mathrm{a} \\
(0.09) \\
\end{array}$ & $\begin{array}{l}0.067 \mathrm{c} \\
(0.025) \\
\end{array}$ & $\begin{array}{l}2.98 \mathrm{c} \\
(0.30)\end{array}$ & $\begin{array}{l}17.67 \mathrm{c} \\
(2.00)\end{array}$ & $\begin{array}{l}94.67 \mathrm{~b} \\
(1.93) \\
\end{array}$ \\
\hline \multirow[t]{3}{*}{$\begin{array}{l}\text { Die L/D } \\
(-)\end{array}$} & 3.4 & $\begin{array}{l}1.16 \mathrm{a} \\
(0.06)\end{array}$ & $\begin{array}{l}0.97 \mathrm{a} \\
(0.10)\end{array}$ & $\begin{array}{l}0.073 a \\
(0.022)\end{array}$ & $\begin{array}{l}2.85 \mathrm{a} \\
(0.28)\end{array}$ & $\begin{array}{l}19.55 \mathrm{a} \\
(2.72)\end{array}$ & $\begin{array}{l}94.36 \mathrm{a} \\
(3.52)\end{array}$ \\
\hline & 6.6 & $\begin{array}{l}1.16 \mathrm{a} \\
(0.06)\end{array}$ & $\begin{array}{c}0.97 \mathrm{a} \\
(0.08)\end{array}$ & $\begin{array}{l}0.077 \mathrm{~b} \\
(0.020)\end{array}$ & $\begin{array}{l}2.98 \mathrm{~b} \\
(0.30)\end{array}$ & $\begin{array}{l}17.86 \mathrm{~b} \\
(2.47)\end{array}$ & $\begin{array}{l}94.23 \mathrm{a} \\
(2.03)\end{array}$ \\
\hline & 9.2 & $\begin{array}{l}1.24 b \\
(0.08)\end{array}$ & $\begin{array}{l}1.00 \mathrm{~b} \\
(0.10)\end{array}$ & $\begin{array}{l}0.065 \mathrm{c} \\
(0.015)\end{array}$ & $\begin{array}{l}2.96 \mathrm{~b} \\
(0.33)\end{array}$ & $\begin{array}{l}17.52 \mathrm{c} \\
(1.93)\end{array}$ & - \\
\hline
\end{tabular}

* Means followed by similar letters within each dependent variable are not significantly different $(\mathrm{P}>0.05$, LSD). Values in parentheses are standard deviation. ER is expansion ratio, UD is unit density, WAI is water absorption index, WSI is water solubility index, PDI is pellet durability index, die L/D is length-to-diameter ratio of the die.

Table 4. Main effects on extrudate physical properties using starch source as a blocking variable $(n=3, \alpha=0.05)$. $^{*}$

\begin{tabular}{|c|c|c|c|c|c|c|c|c|c|c|c|c|c|}
\hline \multirow[b]{2}{*}{ Parameter } & & Hylon VII & Waxy 1 & Hylon VII & Waxy I & Hylon VII & Waxy I & Hylon VII & Waxy I & Hylon VII & Waxy 1 & Hylon VII & Waxy 1 \\
\hline & Levels & \multicolumn{2}{|c|}{ 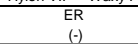 } & \multicolumn{2}{|c|}{$\begin{array}{l}\text { UD } \\
\left(\mathrm{kg} / \mathrm{m}^{3}\right)\end{array}$} & \multicolumn{2}{|c|}{$\begin{array}{l}\text { SV } \\
(\mathrm{m} / \mathrm{s})\end{array}$} & \multicolumn{2}{|c|}{$\begin{array}{c}\text { WAI } \\
(-)\end{array}$} & \multicolumn{2}{|c|}{$\begin{array}{c}\text { WSI } \\
(-)\end{array}$} & \multicolumn{2}{|c|}{$\begin{array}{l}\text { PDI } \\
(\%)\end{array}$} \\
\hline \multirow[t]{3}{*}{$\begin{array}{l}\text { Moisture content } \\
(\% \mathrm{db})\end{array}$} & 15 & $\begin{array}{l}1.21 \mathrm{ax} \\
(0.05)\end{array}$ & $\begin{array}{l}1.24 a y \\
(0.06)\end{array}$ & $\begin{array}{l}1.02 \mathrm{ax} \\
(0.05)\end{array}$ & $\begin{array}{l}1.03 a x \\
(0.06)\end{array}$ & $\begin{array}{c}0.081 \mathrm{ax} \\
(0.01)\end{array}$ & $\begin{array}{c}0.080 a x \\
(0.01)\end{array}$ & $\begin{array}{l}2.999 a x \\
(0.26)\end{array}$ & $\begin{array}{l}2.73 a y \\
(0.27)\end{array}$ & $\begin{array}{c}18.59 \mathrm{ax} \\
(1.93)\end{array}$ & $\begin{array}{c}18.25 \mathrm{ax} \\
(2.23)\end{array}$ & $\begin{array}{c}1.81 \mathrm{ax} \\
(5.88)\end{array}$ & $\begin{array}{c}95.95 a y \\
(1.13)\end{array}$ \\
\hline & 25 & $\begin{array}{l}1.17 \mathrm{bx} \\
(0.04)\end{array}$ & $\begin{array}{l}1.22 \mathrm{by} \\
(0.09)\end{array}$ & $\begin{array}{l}0.94 \mathrm{cx} \\
(0.08)\end{array}$ & $\begin{array}{l}0.944 x \\
(0.13)\end{array}$ & $\begin{array}{c}0.075 \mathrm{bx} \\
(0.01)\end{array}$ & $\begin{array}{c}0.055 \mathrm{by} \\
(0.03)\end{array}$ & $\begin{array}{l}3.05 \mathrm{bx} \\
(0.27)\end{array}$ & $\begin{array}{l}2.79 \text { by } \\
(0.29)\end{array}$ & $\begin{array}{c}18.03 \mathrm{bx} \\
(3.12)\end{array}$ & $\begin{array}{c}17.28 \mathrm{bx} \\
(2.99)\end{array}$ & $\begin{array}{c}91.92 \mathrm{ax} \\
(1.40)\end{array}$ & $\begin{array}{c}94.80 \text { by } \\
(2.04)\end{array}$ \\
\hline & 35 & $\begin{array}{l}1.12 \mathrm{cx} \\
(0.04)\end{array}$ & $\begin{array}{l}1.15 \mathrm{cy} \\
(0.08)\end{array}$ & $\begin{array}{l}0.96 \mathrm{bx} \\
(0.10)\end{array}$ & $\begin{array}{l}0.99 \mathrm{bx} \\
(0.10)\end{array}$ & $\begin{array}{c}0.071 \mathrm{cx} \\
(0.01)\end{array}$ & $\begin{array}{c}0.067 \mathrm{cx} \\
(0.02)\end{array}$ & $\begin{array}{l}3.07 \mathrm{bx} \\
(0.33)\end{array}$ & $\begin{array}{l}2.97 \mathrm{cy} \\
(0.28)\end{array}$ & $\begin{array}{c}17.88 \mathrm{bx} \\
(2.49)\end{array}$ & $\begin{array}{c}19.25 \mathrm{cy} \\
(1.68)\end{array}$ & $\begin{array}{c}94.36 \mathrm{bx} \\
(2.00)\end{array}$ & $\begin{array}{c}96.91 \mathrm{cy} \\
(0.98)\end{array}$ \\
\hline \multirow[t]{3}{*}{$\begin{array}{l}\text { Temperature die } \\
\left({ }^{\circ} \mathrm{C}\right)\end{array}$} & 100 & $\begin{array}{l}1.18 \mathrm{ax} \\
(0.06)\end{array}$ & $\begin{array}{l}1.17 a x \\
(0.08)\end{array}$ & $\begin{array}{l}1.01 \mathrm{ax} \\
(0.08)\end{array}$ & $\begin{array}{l}1.07 \text { ay } \\
(0.07)\end{array}$ & $\begin{array}{c}0.083 a x \\
(0.01)\end{array}$ & $\begin{array}{c}0.081 a x \\
(0.01)\end{array}$ & $\begin{array}{l}2.97 \mathrm{ax} \\
(0.22)\end{array}$ & $\begin{array}{l}2.78 \mathrm{ay} \\
(0.26)\end{array}$ & $\begin{array}{c}18.26 \mathrm{ax} \\
(2.23)\end{array}$ & $\begin{array}{c}17.96 \mathrm{acx} \\
(2.44)\end{array}$ & \begin{tabular}{|c|}
$93.75 a \mathrm{ax}$ \\
$(2.56)$
\end{tabular} & $\begin{array}{c}96.90 \text { ay } \\
(1.42)\end{array}$ \\
\hline & 120 & $\begin{array}{l}1.17 \mathrm{bx} \\
(0.05)\end{array}$ & $\begin{array}{l}1.22 \mathrm{by} \\
(0.09)\end{array}$ & $\begin{array}{l}0.98 \mathrm{bx} \\
(0.08)\end{array}$ & $\begin{array}{l}0.98 \mathrm{bx} \\
(0.07)\end{array}$ & $\begin{array}{c}0.076 \mathrm{bx} \\
(0.01)\end{array}$ & $\begin{array}{c}0.070 \mathrm{by} \\
(0.02)\end{array}$ & $\begin{array}{l}3.03 \mathrm{bx} \\
(0.30)\end{array}$ & $\begin{array}{l}2.75 a y \\
(0.24)\end{array}$ & $\begin{array}{c}18.46 \mathrm{ax} \\
(2.55)\end{array}$ & $\begin{array}{c}18.77 \mathrm{bx} \\
(2.59)\end{array}$ & $\begin{array}{c}93.79 \mathrm{ax} \\
(1.54)\end{array}$ & $\begin{array}{c}95.81 \text { by } \\
(1.49)\end{array}$ \\
\hline & 140 & $\begin{array}{l}1.16 \mathrm{cx} \\
(0.05)\end{array}$ & $\begin{array}{l}1.22 \mathrm{by} \\
(0.08)\end{array}$ & $\begin{array}{l}0.93 c x \\
(0.07)\end{array}$ & $\begin{array}{l}0.91 c x \\
(0.10)\end{array}$ & $\begin{array}{c}0.070 \mathrm{cx} \\
(0.02)\end{array}$ & $\begin{array}{c}0.052 \mathrm{cy} \\
(0.03)\end{array}$ & $\begin{array}{l}3.111 \mathrm{x} x \\
(0.32)\end{array}$ & $\begin{array}{l}2.966 \mathrm{by} \\
(0.34)\end{array}$ & $\begin{array}{c}17.79 \mathrm{bx} \\
(2.84)\end{array}$ & $\begin{array}{c}18.08 \mathrm{acx} \\
(2.33)\end{array}$ & $\begin{array}{c}89.89 \mathrm{bx} \\
(5.32)\end{array}$ & $\begin{array}{c}95.29 \mathrm{cy} \\
(1.63)\end{array}$ \\
\hline \multirow[t]{3}{*}{$\begin{array}{l}\text { Screw speed } \\
\text { (rpm) }\end{array}$} & 100 & $\begin{array}{l}1.16 \mathrm{ax} \\
(0.05)\end{array}$ & $\begin{array}{l}1.19 a y \\
(0.08)\end{array}$ & $\begin{array}{c}0.97 \mathrm{abcx} \\
(0.09)\end{array}$ & $\begin{array}{l}1.00 a x \\
(0.10)\end{array}$ & $\begin{array}{c}0.075 a x \\
(0.01)\end{array}$ & $\begin{array}{c}0.071 \mathrm{ax} \\
(0.02)\end{array}$ & $\begin{array}{l}3.06 \mathrm{ax} \\
(0.30)\end{array}$ & $\begin{array}{l}2.82 \text { ay } \\
(0.30)\end{array}$ & $\begin{array}{c}18.36 \mathrm{ax} \\
(3.01)\end{array}$ & $\begin{array}{c}18.86 \mathrm{ax} \\
(2.63)\end{array}$ & $\begin{array}{c}91.70 \mathrm{ax} \\
(4.94)\end{array}$ & $\begin{array}{c}96.43 a y \\
(1.31)\end{array}$ \\
\hline & 130 & $\begin{array}{l}1.17 \mathrm{bx} \\
(0.06)\end{array}$ & $\begin{array}{l}1.21 \text { by } \\
(0.08)\end{array}$ & \begin{tabular}{|c}
$0.97 \mathrm{abx}$ \\
$(0.08)$
\end{tabular} & $\begin{array}{l}0.99 \mathrm{ax} \\
(0.10)\end{array}$ & $\begin{array}{c}0.077 \mathrm{bx} \\
(0.01)\end{array}$ & $\begin{array}{c}0.072 a x \\
(0.02)\end{array}$ & $\begin{array}{l}2.94 \mathrm{bx} \\
(0.28)\end{array}$ & $\begin{array}{l}2.84 \mathrm{ax} \\
(0.32)\end{array}$ & $\begin{array}{c}18.35 \mathrm{ax} \\
(2.79)\end{array}$ & $\begin{array}{c}18.34 \mathrm{abx} \\
(2.24)\end{array}$ & $\begin{array}{c}93.21 \mathrm{bx} \\
(2.39)\end{array}$ & $\begin{array}{c}96.06 \text { by } \\
(1.74)\end{array}$ \\
\hline & 160 & $\begin{array}{l}1.17 \mathrm{bx} \\
(0.06)\end{array}$ & $\begin{array}{l}1.22 \mathrm{cy} \\
(0.10)\end{array}$ & \begin{tabular}{|c}
$0.98 \mathrm{acx}$ \\
$(0.08)$
\end{tabular} & $\begin{array}{l}0.96 \mathrm{bx} \\
(0.11)\end{array}$ & $\begin{array}{c}0.075 \mathrm{ax} \\
(0.02)\end{array}$ & $\begin{array}{c}0.060 \mathrm{by} \\
(0.03)\end{array}$ & $\begin{array}{l}3.13 c x \\
(0.25)\end{array}$ & $\begin{array}{l}2.84 a y \\
(0.28)\end{array}$ & $\begin{array}{c}17.75 \mathrm{bx} \\
(1.53)\end{array}$ & $\begin{array}{c}17.60 \mathrm{cx} \\
(2.28)\end{array}$ & $\begin{array}{c}93.89 \mathrm{cx} \\
(1.81)\end{array}$ & $\begin{array}{c}95.45 c y \\
(1.74)\end{array}$ \\
\hline \multirow[t]{3}{*}{$\begin{array}{l}\text { Die L/D } \\
(-)\end{array}$} & 3.4 & $\begin{array}{l}1.14 \mathrm{ax} \\
(0.05)\end{array}$ & $\begin{array}{l}\begin{array}{l}1.18 a y \\
(0.07)\end{array} \\
\text { ( }\end{array}$ & $\begin{array}{l}0.96 \mathrm{ax} \\
(0.09)\end{array}$ & $\begin{array}{l}0.97 \mathrm{ax} \\
(0.11)\end{array}$ & $\begin{array}{c}0.077 \mathrm{ax} \\
(0.02)\end{array}$ & $\begin{array}{c}0.070 \mathrm{ax} \\
(0.03)\end{array}$ & $\begin{array}{l}2.81 \mathrm{ax} \\
(0.26)\end{array}$ & $\begin{array}{l}2.89 \mathrm{ax} \\
(0.30)\end{array}$ & \begin{tabular}{|c|}
$19.46 \mathrm{ax}$ \\
$(2.89)$
\end{tabular} & $\begin{array}{c}19.67 a x \\
(2.54)\end{array}$ & $\begin{array}{c}2.71 \mathrm{ax} \\
(4.04)\end{array}$ & $\begin{array}{c}96.01 \text { ay } \\
(1.77)\end{array}$ \\
\hline & 6.6 & $\begin{array}{l}1.15 \mathrm{bx} \\
(0.05)\end{array}$ & $\begin{array}{l}1.17 \mathrm{ax} \\
(0.06)\end{array}$ & $\begin{array}{l}0.95 \mathrm{ax} \\
(0.07)\end{array}$ & $\begin{array}{l}0.98 \mathrm{ay} \\
(0.10)\end{array}$ & $\begin{array}{c}0.083 \mathrm{bx} \\
(0.01)\end{array}$ & $\begin{array}{c}0.072 a y \\
(0.03)\end{array}$ & $\begin{array}{l}3.14 \mathrm{bx} \\
(0.27)\end{array}$ & $\begin{array}{l}2.82 \mathrm{by} \\
(1.60)\end{array}$ & $\begin{array}{c}17.59 \mathrm{bx} \\
(2.50)\end{array}$ & $\begin{array}{c}18.13 \mathrm{bx} \\
(2.42)\end{array}$ & $\begin{array}{c}93.19 \mathrm{bx} \\
(1.79)\end{array}$ & $\begin{array}{c}95.81 \text { ay } \\
(1.05)\end{array}$ \\
\hline & 9.2 & $\begin{array}{l}1.21 c x \\
(0.06) \\
\end{array}$ & $\begin{array}{l}1.27 \text { by } \\
(0.09)\end{array}$ & $\begin{array}{l}1.01 \mathrm{bx} \\
(0.09)\end{array}$ & $\begin{array}{l}1.00 \mathrm{bx} x \\
(0.10)\end{array}$ & $\begin{array}{c}0.068 \mathrm{cx} \\
(0.01)\end{array}$ & $0.062 b y$ & $\begin{array}{l}3.13 \mathrm{bx} \\
(0.21) \\
\end{array}$ & $\begin{array}{l}2.80 \mathrm{by} y \\
(0.34)\end{array}$ & $\begin{array}{c}17.66 \mathrm{bx} \\
(1.86)\end{array}$ & $\begin{array}{c}17.39 \mathrm{cx} \\
(2.01)\end{array}$ & & \\
\hline
\end{tabular}

* Means within a column followed by similar letters $(\mathrm{a}, \mathrm{b}, \mathrm{c})$ for a given dependent variable are not significantly different $(\mathrm{P}>0.05)$ for that independent variable. Means within a row followed by similar letters ( $\mathrm{x}$ or $\mathrm{y})$ for a given dependent variable are not significantly different $(\mathrm{P}>0.05)$ due to starch source. Values in parentheses are standard deviation. ER is expansion ratio, UD is unit density, WAI is water absorption index, WSI is water solubility index, PDI is pellet durability index, L/D is length-to-diameter ratio of the die. 
Table 5. Interaction effects due to starch source, moisture content, die temperature, screw speed and die L/D on extrudate physical properties ( $\mathrm{p}$ values).*

\begin{tabular}{|c|c|c|c|c|c|c|}
\hline $\begin{array}{l}\text { Independent variables and } \\
\text { interactions }\end{array}$ & $\begin{array}{r}\text { ER } \\
(-) \\
\end{array}$ & $\begin{array}{l}\text { UD } \\
\left(\mathrm{kg} / \mathrm{m}^{3}\right)\end{array}$ & $\begin{array}{c}\mathrm{SV} \\
(\mathrm{m} / \mathrm{s})\end{array}$ & $\begin{array}{l}\text { WAI } \\
(-)\end{array}$ & $\begin{array}{l}\text { WSI } \\
(\%)\end{array}$ & $\begin{array}{l}\text { PDI } \\
(\%)\end{array}$ \\
\hline Starch & $<.0001$ & 0.0003 & $<.0001$ & $<.0001$ & 0.7628 & $<.0001$ \\
\hline MC & $<.0001$ & $<.0001$ & $<.0001$ & $<.0001$ & $<.0001$ & $<.0001$ \\
\hline Tdie & $<.0001$ & $<.0001$ & $<.0001$ & $<.0001$ & $<.0001$ & $<.0001$ \\
\hline Speed & $<.0001$ & 0.1904 & $<.0001$ & 0.0037 & $<.0001$ & $<.0001$ \\
\hline L/D die & $<.0001$ & $<.0001$ & $<.0001$ & $<.0001$ & $<.0001$ & 0.1173 \\
\hline Starch*MC & 0.0011 & 0.0709 & $<.0001$ & $<.0001$ & $<.0001$ & $<.0001$ \\
\hline Starch*Tdie & $<.0001$ & $<.0001$ & $<.0001$ & 0.0041 & $<.0001$ & $<.0001$ \\
\hline Starch*Speed & 0.1294 & $<.0001$ & $<.0001$ & $<.0001$ & $<.0001$ & $<.0001$ \\
\hline Starch*L/D die & $<.0001$ & 0.0051 & $<.0001$ & $<.0001$ & 0.0015 & 0.0002 \\
\hline MC*Tdie & $<.0001$ & $<.0001$ & $<.0001$ & 0.0025 & $<.0001$ & $<.0001$ \\
\hline MC*Speed & $<.0001$ & 0.0015 & $<.0001$ & 0.0041 & 0.0003 & $<.0001$ \\
\hline $\mathrm{MC} * \mathrm{~L} / \mathrm{D}$ die & 0.0061 & $<.0001$ & $<.0001$ & $<.0001$ & 0.0002 & $<.0001$ \\
\hline Tdie*Speed & 0.9351 & 0.5716 & $<.0001$ & 0.0001 & $<.0001$ & $<.0001$ \\
\hline Tdie*L/D die & $<.0001$ & $<.0001$ & 0.3124 & $<.0001$ & $<.0001$ & 0.4163 \\
\hline Speed*L/D die & 0.5090 & 0.3242 & $<.0001$ & 0.0004 & $<.0001$ & 0.0007 \\
\hline Starch*MC*Tdie & $<.0001$ & $<.0001$ & $<.0001$ & $<.0001$ & 0.0194 & $<.0001$ \\
\hline Starch*MC*Speed & 0.0006 & 0.2487 & $<.0001$ & $<.0001$ & $<.0001$ & $<.0001$ \\
\hline Starch*MC* L/D die & $<.0001$ & 0.0011 & $<.0001$ & 0.0012 & $<.0001$ & . \\
\hline Starch*Tdie*Speed & 0.3710 & 0.0013 & $<.0001$ & 0.0661 & $<.0001$ & $<.0001$ \\
\hline Starch*Tdie*L/D die & 0.0008 & 0.0346 & $<.0001$ & 0.5730 & $<.0001$ & . \\
\hline Starch*Speed*L/D die & 0.1755 & $<.0001$ & $<.0001$ & 0.0158 & $<.0001$ & . \\
\hline MC*Tdie*Speed & 0.1539 & 0.0302 & $<.0001$ & $<.0001$ & $<.0001$ & $<.0001$ \\
\hline MC*Tdie*L/D die & 0.0553 & $<.0001$ & $<.0001$ & $<.0001$ & $<.0001$ & $<.0001$ \\
\hline MC*Speed*L/D die & 0.0003 & 0.2030 & $<.0001$ & 0.0263 & $<.0001$ & 0.8807 \\
\hline Tdie*Speed*L/D die & 0.2703 & 0.0472 & $<.0001$ & 0.0009 & $<.0001$ & 0.0013 \\
\hline Starch*MC*Tdie*Speed & 0.1026 & 0.0401 & $<.0001$ & 0.0038 & $<.0001$ & $<.0001$ \\
\hline Starch*MC*Tdie*L/D die & 0.0156 & $<.0001$ & $<.0001$ & $<.0001$ & $<.0001$ & . \\
\hline Starch*MC*Speed*L/D die & 0.0446 & 0.0003 & $<.0001$ & $<.0001$ & $<.0001$ & . \\
\hline Starch*Tdie*Speed $*$ L/D die & 0.0413 & 0.0205 & $<.0001$ & 0.0012 & $<.0001$ & . \\
\hline MC*Tdie*Speed*L/D die & 0.1373 & 0.0002 & $<.0001$ & $<.0001$ & $<.0001$ & 0.4805 \\
\hline Starch*MC*Tdie*Speed*L/D die & 0.0036 & $<.0001$ & $<.0001$ & $<.0001$ & $<.0001$ & \\
\hline
\end{tabular}

* MC is moisture content, Tdie is temperature of the die, ER is expansion ratio, UD is unit density, WAI is water absorption index, WSI is water solubility index, PDI is pellet durability index, L/D is length-to-diameter ratio of the die.
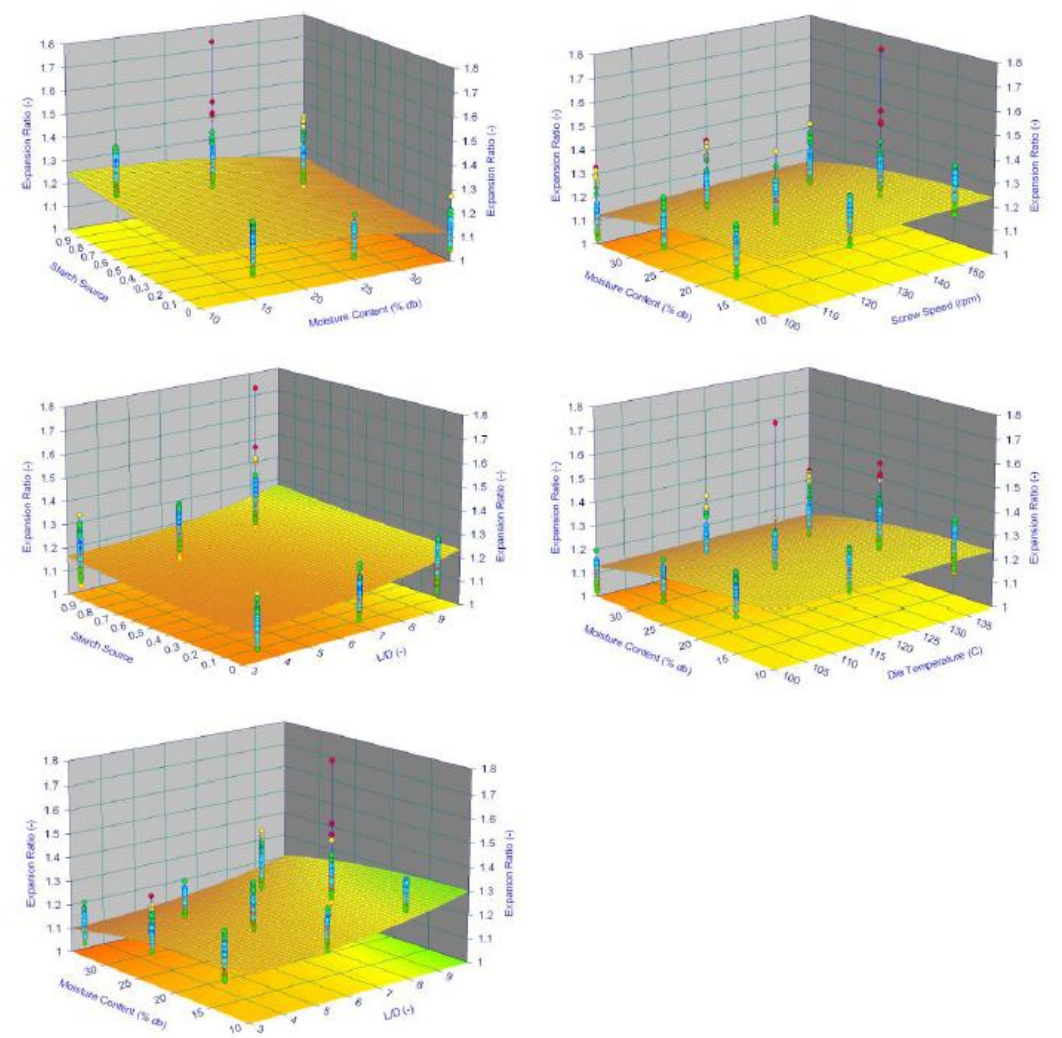

Figure 1. Treatment combination effects on expansion ratio. (Starch source $0=0 \%$ amylopectin, $1=100 \%$ amylopectin). 

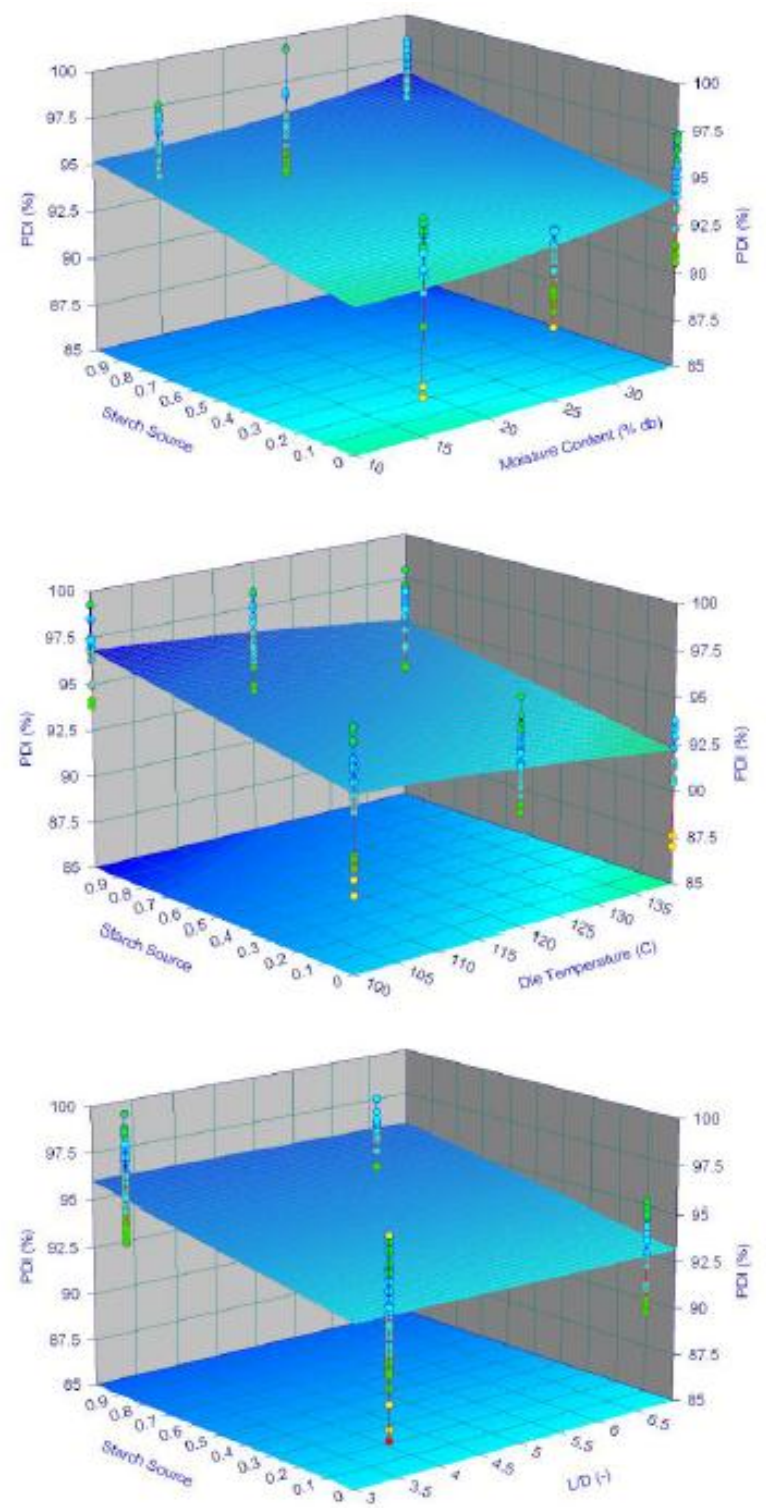

Figure 2. Treatment combination effects on pellet durability index (PDI). (Starch source $0=0 \%$ amylopectin, 1 $=100 \%$ amylopectin).

\subsection{Extrudate Properties}

Expansion ratio (ER)

Expansion ratio is a decisive factor for aqua feeds. The degree of expansion upon exiting the die impacts the unit density, and thus the floatability of the feed. Fragility and hardness of extrudates are also affected by ER (Kannadhason et al., 2009a, Rosentrater et al., 2009a).

The proportion of amylopectin in starch and the feed moisture content mainly affect expansion. Studies on the effects of different starch sources on DDGS-based extrudates showed increasing expansion for blends with higher amylopectin proportions of the starch (Kannadhason et al., 2009a). Amylopectin is considered to have swelling properties whereas amylose acts as a diluent (Tester and Morrison, 1990). Regarding the main treatment effects (Table 3), the impact of higher amylopectin portion was detected by a $2.5 \%$ increase of ER when using the blend with the $100 \%$ amylopectin proportion compared to the one with $30 \%$. Investigating different starch sources, the highest expansion ratio was observed at 1.65 when using cassava starch (83\% amylopectin) in combination with 20\% DDGS, whereas the lowest ER at 1.09 had been recorded for the $72 \%$ amylopectin corn starch in combination with 40\% DDGS (Kannadhason et al., 2009a).

Feed moisture is a key factor in extrusion processing which highly impacts expansion and density. Due to changes in the molecular structure of amylopectin, moisture reduces the melt elasticity and expansion decreases 
whereas density increases (Ding et al., 2005). This could be observed in the main treatment effects (Table 3) where an increase in moisture content from 15 to $35 \%$ yielded a $6.6 \%$ decrease in ER from 1.22 to 1.14. The blends with the Hylon VII as the starch source reached significantly higher ER than the Waxy I (100\% amylopectin) blends at the same MC level (Table 4).

During gelatinization, the crystalline structure of the starch granules is destroyed and the amylopectin turns into an amorphous state. The starch granules form a gel that is more expanded than their initial state. At the same time the amylose (if present) leaches out of the amylopectin and forms a continuous gel phase outside the granules (Hermansson and Svegmark, 1996; Yu and Christie, 2005). Shear forces and temperature affect the gel formation. However, different starch sources show different optimum temperatures for expansion (Chinnaswamy and Hanna, 1988). For the main treatment effects, a small but significant increase in ER from 1.17 to 1.20 was observed when increasing the screw speed from 100 to $160 \mathrm{rpm}$ (Table 3). Increasing the temperature from 100 to $120^{\circ} \mathrm{C}$ yielded also a small but significant increase in ER from 1.17 to 1.19 . The blends with Hylon VII resulted in significantly lower ER with increasing temperature whereas the Waxy I (100\% amylopectin) blends increased in ER with increasing temperature only from 100 to $120^{\circ} \mathrm{C}$. The Waxy I blends reached significantly higher ER than the Hylon VII blends. For both blends ER increased significantly with increasing screw speed. Raising the screw speed from 130 to 160 resulted in no significant difference in ER for the Hylon VII blends (Table 4). The highest ER for Hylon VII and Waxy I were found at 1.31 and 1.43 for the treatment combination at $15 \% \mathrm{MC}, 120^{\circ} \mathrm{C}, 160 \mathrm{rpm}$ and $9.2 \mathrm{~L} / \mathrm{D}$ ratio of the die and $25 \% \mathrm{MC}, 140^{\circ} \mathrm{C}, 160 \mathrm{rpm}$, and $6.6 \mathrm{~L} / \mathrm{D}$ ratio of the die, respectively.

Radial expansion was calculated as the ratio between the diameter of the extrudate and the die, after extrusion and drying. Longitudinal and volumetric expansion were neglected. For all blends, ER did not change when the die L/D ratio increased from 3.4 to 6.6, whereas expansion ratio significantly increased by $6.9 \%$ with an increase in die L/D ratio (decrease in die diameter) from 6.6 to 9.2 (Table 3). Again, ER for the Waxy 1 blends was significantly higher than the Hylon VII blends (Table 4). Several interactions existed between independent variables (Table 4). Regarding the treatment combination effects, ER decreased with higher moisture content in combination with lower amylopectin content, lower screw speed, lower temperature setting, and lower L/D ratio, respectively. Figure 1 shows these behaviours, and indicates non-linear relationships between these variables. The highest ER was found at 1.43 with the $100 \%$ amylopectin starch, containing $35 \%$ feed moisture content, at highest temperature setting $\left(140^{\circ} \mathrm{C}\right)$, highest screw speed and highest L/D ratio of the die. The lowest ER was detected at 1.05 for the same blend but with $25 \%$ feed moisture content, lowest temperature setting $\left(100^{\circ} \mathrm{C}\right)$, lowest screw speed (100 rpm) and lowest L/D ratio of the die (3.4). Also, ER significantly increased with higher screw speed. This is in line with the assumption that increased shear leads to higher expansion due to increased gelatinization unless a certain shear degree is reached. Both, die nozzle diameter and length determine an increase or decrease in back pressure, residence time and shear as the dough passes the die section. This produces a sudden drop from high pressure to atmospheric pressure impacting the strength of water flashing-off at the die and applying enormous tensile forces while the melt expands. Forces and temperature drop lead to changes in the material states. For fully expansion an optimum amount of pressure is required which is achieved by an optimum die L/D ratio (Ganjyal and Hanna, 2007).

Unit density (UD)

Unit density determines the density for a single extrudate. Generally, it is inversely related to the expansion ratio. Unit density can predict the floatability of extrudates which is mandatory for top feeding fish, as Nile tilapia, to ensure food supply and reduce feed loss and water pollution.

All main treatment effects significantly affected the unit density, except for screw speed and die L/D ratio where at least two treatments did not show significant differences. Changes due to the starch source were small but significant. The Hylon VII blends had mostly lower UD values than the Waxy I (100\% amylopectin) blends. Initially, UD declined from 1.02-0.94 kg/m $\mathrm{m}^{3}$ when increasing MC from $15-25 \%$, but then increased significantly when enhancing the MC from 25-35\% (Table 3 and Table 4). These differences do not conform with the changes in ER what may be related to the higher standard deviation of UD. Increasing the temperature from $100-140^{\circ} \mathrm{C}$ yielded a significant reduction in UD by $11.5 \%$ (Table 3). This agrees with Ding et al. (2005) findings that feed moisture and temperature significantly affect extrudate density.

The highest UD was recorded at $1.18 \mathrm{~kg} / \mathrm{m}^{3}$ for the treatment combination with Waxy $\mathrm{I}$, at highest MC (35\%), lowest temperature setting $\left(100^{\circ} \mathrm{C}\right)$, highest die L/D ratio of the die $(9.2)$ and medium screw speed $(130 \mathrm{rpm})$. The lowest UD was also observed for the high amylopectin blends at $0.76 \mathrm{~kg} / \mathrm{m}^{3}$ at medium MC $(25 \%)$, highest temperature $\left(140^{\circ} \mathrm{C}\right)$, highest screw speed $(160 \mathrm{rpm})$ and highest $\mathrm{L} / \mathrm{D}$ ratio of the die (9.2) setting. For the same 
treatment combination as the lowest UD was determined, the highest expansion ratio was observed. Blends with high amylopectin content starch sources in combination with appropriate moisture content, temperature, and screw speed, resulted in increased gel formation and thus higher expansion. As anticipated, the significance of higher temperature resulting in lower unit density was evident in this study. These findings are in complete agreement with the results of Kannadhason et al. (2009b) and Rosentrater et al. (2009a). An inverse relationship between UD and ER was not clearly detected which could be ascribed to the relatively high standard deviation of the UD data.

Sinking velocity (SV)

The sinking velocity relates to the stability and floatability of extrudates. It can conclude about the porosity of an extrudate and how fast it absorbs water and sinks. Therefore, sinking velocity is related to the unit density, expansion ratio and the biochemical and biomechanical changes that occur inside the extruder barrel. All main treatment effects showed significant differences in SV (Table 3). Regarding the main effects, the blend containing Waxy I as a starch source had $10.5 \%$ significantly lower SV than the Hylon VII blends. No clear pattern could be observed on changes in SV due to moisture content, screw speed, and die L/D ratio. Increasing the processing temperature from 100 to $140^{\circ} \mathrm{C}$ yielded a significant decrease in SV by $25.6 \%$, which is in line with the findings of Kannadhason et al. (2009b) and Rosentrater et al. (2009a, 2009b). Changes in SV are only related to changes in UD for moisture content and temperature. Increasing the screw speed from 130 to $160 \mathrm{rpm}$ resulted in a 9.5\% significant increase in SV, whereas the increase in screw speed from 100 to $130 \mathrm{rpm}$ yielded a decrease of $1.4 \%$. The slowest SV value was observed at $0.002 \mathrm{~m} / \mathrm{s}$ for the Waxy I diet containing at $25 \%$ moisture content, highest temperature $\left(140^{\circ} \mathrm{C}\right)$, highest screw speed setting $(160 \mathrm{rpm})$ and highest $\mathrm{L} / \mathrm{D}$ ratio of the die (9.2). This setting complies with the treatment combination, which had the highest expansion and evidently leading to higher floatability. The same observation was made by Kannadhason et al. (2009a) and Rosentrater et al. (2009a). Unexpectedly, the fastest SV at $0.102 \mathrm{~m} / \mathrm{s}$ could also be observed at the settings as for the fastest SV but at medium screw speed (130 rpm), and lowest die L/D ratio (3.4). Interaction effects among all independent variables were observed, except for temperature and L/D. This showed the big impact of screw speed on SV.

\section{Water absorption and water solubility index (WAI and WSI)}

Materials based on starch absorb water due to the abundance of hydroxyl group forming hydrogen bonds with water. Gelatinization involves the destruction of the crystalline-like structure of starch (Pizzoferrato et al., 1999) and expands the water absorption and water solubility properties (Colonna and Mercier, 1983).

The water absorption index reflects the weight of gel that is retained per unit weight of dry sample, whereas the water solubility index describes the percent of dry sample of the supernatant (Anderson et al., 1969). Both properties depend on the amylose-to-amylopectin ratio, moisture content, screw speed, and die geometry (Colonna and Mercier, 1983), which was observed in this study. However, the main treatment effects showed a significant increase of $6.9 \%$ in WAI when raising the amylopectin ratio from 30 to $100 \%$, whereas the WSI yielded no significant difference with changes in starch source. Similar results were reported by Mani and Bhattacharya for WAI (1998). The increase in WAI with higher amylopectin ratio can be ascribed to the escalating gelatinization and debranching of the amylopectin structure yielding an expanded matrix capable of holding more water. Other than the starch source, feed moisture, and temperature had significant effects on WAI, which increased with higher moisture content and temperature. This can be explained by the gel-forming capacity of macromolecules and the water-binding capacity of hydrophilic groups for gelatinized corn starch (Gomez and Aguilera, 1983). Regarding the main treatment effects due to the starch source, Hylon VII blends reached significantly higher values than Waxy I (100\% amylopectin) blends (Table 4).

The WAI of the blends decreased with lower temperatures indicating a more dense structure of the extrudate with a lower water holding capacity. The lower WAI values with reducing moisture content can be ascribed to the starch dextrinization that occurs at low-moisture contents below 20\% during high-shear extrusion cooking, whereas gelatinization predominates at moisture contents higher than 20\% (Gomez and Aguilera, 1984). The highest WAI was detected at 3.6 for the treatment combination of Waxy I blends, at $35 \%$ moisture content, $140^{\circ} \mathrm{C}, 130 \mathrm{rpm}$ and $\mathrm{L} / \mathrm{D}$ ratio of the die of 3.4. The lowest WAI was observed at 2.3 for the treatment combination of the Hylon VII blends at $35 \%$ moisture content, $120^{\circ} \mathrm{C}, 130 \mathrm{rpm}$ and L/D ratio of the die of 3.4.

WSI is a measure of starch dextrinization (Bhatnagar and Hanna, 1994) and depends on available solubles that increase with starch degradation (Jin et al., 1995). In contrast to WAI, the starch source showed no significant effect on WSI and with higher screw speeds from 100 to $160 \mathrm{rpm}$ WSI significantly decreased by $5.1 \%$ (Table 3). These unexpected results are also contradictory to other findings where WSI increased with higher screw speed 
starting at 150 and $180 \mathrm{rpm}$, respectively (Gomez and Aguilera, 1983; Jin et al., 1995; Iwe, 1998) and can be ascribed to relatively high standard deviations in this study. They same may apply for changes in L/D of the die from 3.4 to 9.2 where a significant decrease of WSI by $10.4 \%$ occurred. The findings for highest and lowest WSI confirm the assumption that relatively high standard deviations may have impacted the results. The treatment combination with the $100 \%$ amylopectin starch at $25 \%$ moisture content, $120^{\circ} \mathrm{C}, 130 \mathrm{rpm}$ and L/D of 3.4 showed the highest WSI at $24.2 \%$, whereas the lowest WSI was found at $10.75 \%$ for $30 \%$ amylose starch at $35 \%$ moisture content, $120^{\circ} \mathrm{C}, 130 \mathrm{rpm}$ and $\mathrm{L} / \mathrm{D}$ ratio of 3.4.

Pellet durability index (PDI)

Resistance against destructive and abrasive forces during transportation, handling, and storage is a highly desired property of extrudates in order to maintain their value and quality (Rosentrater et al., 2005). Fines, such as dust, will not be consumed by fish and have to be minimized to avoid economic losses and minimize water pollution (Sørensen et al., 2010).

Durability is tested by simulating the mechanical handling of extrudates during tumbling test and is defined by possible fines produced. It is not only dependent on the gelatinization of starch but also on the heat treatment and moisture content of the blends. This is reflected in the main treatment effects of each independent factor on pellet durability index (Table 3). A significant increase in PDI from 92.85 to $95.98 \%$ was observed between Hylon VII and Waxy I (100\% amylopectin) blends. Decreasing the temperature setting from 140 to $100^{\circ} \mathrm{C}$, resulted in an increase of PDI from 92.98 to $95.13 \%$ for all blends. There were no significant differences detected when changing the L/D of the die from 3.4 to 6.4. The treatment combination effects illustrated that PDI steadily increased with higher moisture content and higher amylopectin portion. As shown in Figure 2, the graphs show an increase in PDI with lower temperature and using Waxy I starch (100\% amylopectin). Figure 2 shows these behaviours, and indicates non-linear relationships between these variables. Changes in PDI in combination with starch source and L/D ratio, demonstrated an increase only when the amylopectin content was raised, but not with changes in L/D ratio only. This was also reflected in the main treatment effects. The highest PDI was found at $99.3 \%$ when Waxy I blends were used containing $25 \%$ moisture content, using a $\mathrm{L} / \mathrm{D}$ ratio of 3.4 at $100^{\circ} \mathrm{C}$ and 130 rpm screw speed. The lowest PDI was detected with the Hylon VII starch (30\% amylopectin), with 15\% MC, at $140^{\circ} \mathrm{C}, 100 \mathrm{rpm}$, and $\mathrm{L} / \mathrm{D}$ ratio of 3.4. Overall, all extrudates yielded good pellet durability indices that were significantly affected by the starch source, moisture content, and temperature settings. These observations are in accordance with findings done by Kannadhason et al. (2009a, 2009b) and confirm better stabilizing properties with higher amylopectin portions of starch. Heat treatment and available water also highly affected the gelatinization of starch and thus the cohesion and stability of the extrudates.

\section{Conclusions}

The incorporation of either of two starch sources with varying amylopectin portions in combination with distillers dried grains with solubles, soy, and other ingredients was investigated to determine their effects on resulting binding capacity. Feed ingredients (moisture content and amylopectin-amylose ratio) and processing conditions (die temperature, L/D, and screw speed) were modified to examine their effects on the properties of the resulting extrudates. Altering the amylopectin portions and the starch sources, respectively, had significant effects on all measured parameters. Moisture content, temperature setting, and screw speed, significantly affected most of the extrudate properties, whereas L/D ratio of the die showed only some effects on the resulting extrudates. Unexpectedly, expansion ratio was low. As anticipated, ER increased with higher amylopectin portion and higher screw speed, whereas it declined with higher moisture content. Highest PDI values were achieved by using the starch source with the highest amylopectin portion, highest moisture content, and lowest temperature setting. The starch source with higher amylopectin in combination with adequate moisture content, temperature, and screw speed demonstrated to be the best choice for better quality extrudates in terms of durability and binding capacity.

\section{Acknowledgements}

The authors thank the North Central Agricultural Research Laboratory, USDA-ARS, Brookings, South Dakota, for funding, facilities, equipment, and supplies.

\section{References}

AACC. (2000). Method 44-19, Moisture-air oven method, drying at $135^{\circ} \mathrm{C}$. AACC Approved Methods. $10^{\text {th }}$ Ed. American Association of Cereal Chemists, St. Paul, MN.

Anderson, R. A., Conway, H. F., Pfeifer, V. F., \& Griffin, L. E. J. (1969). Gelatinization of corn grits by roll-and extrusion cooking. Cereal Science Today, 14, 4-7, 11-12. 
ASAE. (2004). Engineering Standards, Practices and Data. American Society of Agricultural and Biological Engineers, St. Joseph, MI. ISBN 1-892769-38-7.

Ayadi, F., Rosentrater, K. A., \& Muthukumarappan, K. (2009). A review of alternative protein sources in aquaculture feeds. ASABE Paper No 1008496. St. Joseph, MI, American Society of Agricultural and Biological Engineers.

Bals, B., Dale, B., \& Balan, V. (2006). Enzymatic hydrolysis of distiller's dry grain and solubles (DDGS) using ammonia fiber expansion pretreatment. Energy and Fuels, 20(6), 2732-2736. http://dx.doi.org/10.1021/ef060299s

Bhatnagar, S., \& Hanna, M. A. (1994). Amylose-lipid complex formation during single-screw extrusion of various corn starches. Cereal Chemistry, 71(6), 582-587.

Cheng, Z. J., \& Hardy, R. W. (2004). Nutritional value of diets containing distiller's dried grain with solubles for rainbow trout, Oncorhynchus mykiss. Journal of Applied Aquaculture, 15(3/4), 101-113. http://dx.doi.org/10.1300/J028v15n03_08

Chevanan, N., Muthukumarappan, K., Rosentrater, K. A., \& Julson, J. L. (2007). Effect of die dimensions on extrusion processing parameters and properties of DDGS-based aquaculture feeds. Cereal Chemistry, 84(4), 389-398. http://dx.doi.org/10.1094/CCHEM-84-4-0389

Chevanan, N., Rosentrater, K. A., \& Muthukumarappan, K. (2008). Effect of DDGS, moisture content, and screw speed on physical properties of extrudates in single-screw extrusion. Cereal Chemistry, 85(2), 132-139. http://dx.doi.org/10.1094/CCHEM-85-2-0132

Chevanan, N., Muthukumarappan, K., \& Rosentrater, K. A. (2009). Extrusion studies of aquaculture feed using distillers dried grains with solubles and whey. Food and Bioprocess Technology, 2(2), 177-185. http://dx.doi.org/10.1007/s11947-007-0036-8

Chevanan, N., Rosentrater, K. A., \& Muthukumarappan, K. (2010). Effects of processing conditions on single screw extrusion of feed ingredients containing DDGS. Food and Bioprocess Technology, 3(1), 111-120. http://dx.doi.org/10.1007/s11947-008-0065-y

Chinnaswamy, R., \& Hanna, M. A. (1988). Relationship between amylose content and extrusion-expansion properties of corn starches. Cereal Chemistry, 65(2), 138-143.

Colonna, P., \& Mercier, C. (1983). Macromolecular modifications of manioc starch components by extrusion-cooking with and without lipids. Carbohydrate Polymers, 3(2), 87-108. http://dx.doi.org/10.1016/0144-8617(83)90001-2

Coyle, S. D., Mengel, G. J., Tidwell, J. H., \& Webster, C. D. (2004). Evaluation of growth, feed utilization, and economics of hybrid tilapia, Oreochromis niloticus x Oreochromis aureus, fed diets containing different protein sources in combination with distillers dried grains with solubles. Aquaculture Research, 35(4), 365-370. http://dx.doi.org/10.1111/j.1365-2109.2004.01023.x

Ding, Q. B., Ainsworth, P., Tucker, G., \& Marson, H. (2005). The effect of extrusion conditions on the physicochemical properties and sensory characteristics of rice-based expanded snacks. Journal of Food Engineering, 66(3), 283-289. http://dx.doi.org/10.1016/j.jfoodeng.2004.03.019

Ganjyal, G. M., \& Hanna, M. A. (2004). Effects of extruder die nozzle dimensions on expansion and micrographic characterization during extrusion of acetylated starch. Starch/Stärke, 56(3-4), 108-117. http://dx.doi.org/10.1002/star.200300200

Gomez, M. H., \& Aguilera, J. M. (1983). Changes in the starch fraction during extrusion-cooking of corn. Journal of Food Science, 48(2), 378-381. http://dx.doi.org/10.1111/j.1365-2621.1983.tb10747.x

Gomez, M. H., \& Aguilera, J. M. (1984). A physicochemical model for extrusion of corn starch. Journal of Food Science, 49(1), 40-43. http://dx.doi.org/10.1111/j.1365-2621.1984.tb13664.x

Hermansson, A. M., \& Svegmark, K. (1996). Developments in the understanding of starch functionality. Trends in Food Science and Technology, 7(11), 345-353. http://dx.doi.org/10.1016/S0924-2244(96)10036-4

Himadri, K. D., Tapani, M. H., Myllymaki, O. M., \& Malkikki, Y. (1993). Effects of formulation and processing variables on dry fish feed pellets containing fish waste. Journal of the Science of Food and Agriculture, 61(2), 181-187. http://dx.doi.org/10.1002/jsfa.2740610208

Iwe, M. O. (1998). Effects of extrusion cooking on functional properties of mixtures of full-fat soy and sweet 
potato. Plant Foods for Human Nutrition, 53(1), 37-46. http://dx.doi.org/10.1023/A:1008095703026

Jacques, K. A., Lyons, T. P., \& Kelsall, D. R. (2003). The Alcohol Textbook $4^{\text {th }}$ ed. p.379. Nottingham, United Kingdom, Nottingham University Press.

Jin, Z., Hsieh, F., \& Huff, H. E. (1995). Effects of soy fiber, salt, sugar and screw speed on physical properties and microstructure of corn meal extrudate. Journal of Cereal Science, 22(2), 185-194. http://dx.doi.org/10.1016/0733-5210(95)90049-7

Kannadhason, S., Muthukumarappan, K., \& Rosentrater, K. A. (2009a). Effect of starch sources and protein content on extruded aquaculture feed containing DDGS. Food and Bioprocess Technology (in press). DOI 10.1007/s11947-008-0177-4. http://dx.doi.org/10.1007/s11947-008-0177-4

Kannadhason, S., Muthukumarappan, K., \& Rosentrater, K. A. (2009b). Effects of ingredients and extrusion parameters on aquafeeds containing DDGS and tapioca starch. Journal of Aquaculture Feed Science and Nutrition, 1(1), 6-21.

Kannadhason, S., Rosentrater, K. A., \& Muthukumarappan, K. (2010). Twin screw extrusion of DDGS-based aquaculture feeds. Journal of the World Aquaculture Society, 41(51), 1-15. http://dx.doi.org/10.1111/j.1749-7345.2009.00328.x

Keetels, C. J. A. M., Oostergetel, G. T., \& van Vliet, T. (1996a). Recrystallization of amylopectin in concentrated starch gels. Carbohydrate Polymers, 30(1), 61-64. http://dx.doi.org/10.1016/S0144-8617(96)00057-4

Keetels, C. J. A. M., van Vliet., T. \& Walstra, P. (1996b). Gelation and retrogradation of concentrated starch $\begin{array}{lllll}\text { systems, } & 1, \quad \text { Gelation. } & \text { Food } & \text { Hydrocolloids, } & \text { 10(3), }\end{array}$ http://dx.doi.org/10.1016/S0268-005X(96)80011-7

Klopfenstein, T. J., Erickson, G. E., \& Bremer, V. R. (2008). Board-invited review, Use of distillers by-products in the beef cattle feeding industry. Journal of Animal Science, 86(5), 1223-1231. http://dx.doi.org/10.2527/jas.2007-0550

Lai, L. S., \& Kokini, J. L. (1991). Physicochemical changes and rheological properties of starch during extrusion (A review). Biotechnology Progress, 7(3), 251-266. http://dx.doi.org/10.1021/bp00009a009

Lim, C., Garcia, J. C. Yildirim-Aksoy, M., Klesius, P. H., Shoemaker, C. A., \& Evans, J. J. (2007). Growth response and resistance to Streptococcus iniae of Nile tilapia, Oreochromis niloticus, fed diets containing distiller's dried grains with solubles. Journal of the World Aquaculture Society, 38(2), 231-237. http://dx.doi.org/10.1111/j.1749-7345.2007.00093.x

Lim, C., Yildirim-Aksoy, M., \& Klesius, P. H. (2009). Growth response and resistance to Edwardsiella ictaluri of channel catfish, Ictalurus punctatus, fed diets containing distiller's dried grains with solubles. Journal of the World Aquaculture Society, 40(2), 182-193. http://dx.doi.org/10.1111/j.1749-7345.2009.00241.x

Liu, X., Yu, L., Liu, H., Chen, L., \& Li, L. (2009). Thermal decomposition of corn starch with different amylose/amylopectin ratios in open and sealed systems. Cereal Chemistry, 86(4), 383-385. http://dx.doi.org/10.1094/CCHEM-86-4-0383

Lumpkins, B. S., Batal, A. B., \& Dale, N. M. (2004). Evaluation of distillers dried grains with solubles as a feed ingredient for broilers. Poultry Science, 83(11), 1891-1896. http://dx.doi.org/10.1093/ps/83.11.1891

Mani, R., \& Bhattacharya, M. (1998). Properties of injection moulded starch/synthetic polymer blends-III. Effect of amylopectin to amylose ratio in starch. European Polymer Journal, 34(10), 1467-1475. http://dx.doi.org/10.1016/S0014-3057(97)00273-5

Manners, D. J. (1989). Recent developments in our understanding of amylopectin structure. Carbohydrate Polymers, 11(2), 87-112. http://dx.doi.org/10.1016/0144-8617(89)90018-0

NSFI. (2008). Hylon VII. Technical service bulletin. Bridgewater, NJ, National Starch and Chemical Company. Available at http://eu.foodinnovation.com/docs/HYLONVII.pdf. Accessed 6 May 2010.

Pizzoferrato, L., Rotilio, G., \& Paci, M. (1999). Modification of structure and digestibility of chestnut starch upon cooking, A solid state 13C CP MAS NMR and enzymatic degradation study. Journal of Agricultural and Food Chemistry, 47(10), 4060-4063. http://dx.doi.org/10.1021/jf9813182

Robinson, E. H., \& Li., M. H. (2008). Replacement of soybean meal in channel catfish, Ictalurus punctatus, diets with cottonseed meal and distiller's dried grains with solubles. Journal of the World Aquaculture Society, 39(4), 521-527. http://dx.doi.org/10.1111/j.1749-7345.2008.00190.x 
Rosentrater, K. A., Richard, T. L., Bern, C. J., \& Flores, R. A. (2005). Small-scale extrusion of corn masa by-products. Cereal Chemistry, 82(4), 436-446. http://dx.doi.org/10.1094/CC-82-0436

Rosentrater, K. A., \& Muthukumarappan, K. (2006). Corn ethanol coproducts, Generation, properties, and future prospects. International Sugar Journal, 108(1295), 648-657.

Rosentrater, K. A., Muthukumarappan, K., \& Kannadhason, S. (2009a). Effects of ingredients and extrusion parameters on aquafeeds containing DDGS and potato starch. Journal of Aquaculture Feed Science and Nutrition, 1(1), 22-38.

Rosentrater, K. A., Muthukumarappan, K., \& Kannadhason, S. (2009b). Effects of ingredients and extrusion parameters on properties of aquafeeds containing DDGS and corn starch. Journal of Aquaculture Feed Science and Nutrition, 1(2), 44-60.

Schingoethe, D. J., Kalscheur, K. F., Hippen, A. R., \& Garcia, A. D. (2009). Invited review, The use of distillers products in dairy cattle diets. Journal of Dairy Science, 92(12), 5802-5813. http://dx.doi.org/10.3168/jds.2009-2549

Sørensen, M., Morken, T., Kosanovic, M., \& Øverland, M. (2010). Pea and wheat starch possess different processing characteristics and affect physical quality and viscosity of extruded feed for Atlantic salmon. Aquaculture Nutrition (in press). http://dx.doi.org/10.1111/j.1365-2095.2010.00767.x

Stein, H. H., \& Shurson, G. C. (2009). Board-invited review, The use and application of distillers dried grains with solubles in swine diets. Journal of Animal Science, 87(4), 1292-1303. http://dx.doi.org/10.2527/jas.2008-1290

Tester, R. F., \& Morrison, W. R. (1990). Swelling and gelatinization of cereal starches. I. Effects of amylopectin, amylose, and lipids. Cereal Chemistry, 67(6), 551-557.

Thiex, N. (2009). Evaluation of analytical methods for the determination of moisture, crude protein, crude fat, and crude fiber in distillers dried grains with solubles. Journal of AOAC International, 92(1), 61-73.

Thompson, K. R., Rawles, S. D., Metts, L. S., Smith, R., Wimsatt, A., Gannam, A. L., Twibell, R. G., Johnson, R. B., Brady, Y. J., \& Webster, C. D. (2008). Digestibility of dry matter, protein, lipid, and organic matter of two fishmeals, two poultry by-product meals, soybean meal, and distiller's dried grains with solubles in partial diets for sunshine bass, Morone chrysops x M. saxatilis. Journal of the World Aquaculture Society, 39(3), 352-363. http://dx.doi.org/10.1111/j.1749-7345.2008.00174.x

Webster, C. D., Tidwell, J. H., \& Goodgame, L. S. (1993). Growth, body composition, and organoleptic evaluation of channel catfish fed diets containing different percentages of distillers' grains with solubles. The Progressive Fish-Culturist, http://dx.doi.org/10.1577/1548-8640(1993)055<0095:GBCAOE>2.3.CO;2

Webster, C. D., Tidwell, J. H., Goodgame-Tiu, L. S., \& Yancey, D. H. (1995). Evaluation of distillers grains with solubles as an alternative plant protein in aquaculture diets. In Nutrition and Utilization Technology in Aquaculture, p.192. C.E. Lim and D.J. Sessa, eds. Champaign, IL: AOCS Press.

Wu, V. W., Rosati, R. R., \& Brown, P. B. (1996). Effect of diets containing various levels of protein and ethanol coproducts from corn on growth of tilapia fry. Journal of Agricultural and Food Chemistry, 44(6), 1491-1493. http://dx.doi.org/10.1021/jf950733g

Yu, L., \& Christie, G. (2005). Microstructure and mechanical properties of orientated thermoplastic starches. Journal of Materials Science, 40(1), 111-116. http://dx.doi.org/10.1007/s10853-005-5694-1

\section{Copyrights}

Copyright for this article is retained by the author(s), with first publication rights granted to the journal.

This is an open-access article distributed under the terms and conditions of the Creative Commons Attribution license (http://creativecommons.org/licenses/by/4.0/). 